\title{
The Effect of Multidomain Interventions on Global Cognition, Symptoms of Depression and Apathy - A Pooled Analysis of Two Randomized Controlled Trials
}

\author{
M.G.H.E. den Brok ${ }^{1,2}$, M.P. Hoevenaar-Blom ${ }^{2}$, N. Coley,5, S. Andrieu ${ }^{5,6}$, J. van Dalen 1,2, Y. Meiller , \\ J. Guillemont ${ }^{5}$, C. Brayne ${ }^{8}$, W.A. van Gool ${ }^{3}$, E.P. Moll van Charante ${ }^{3,4}$, E. Richard ${ }^{1,3}$ On behalf of the preDIVA \\ and MAPT/DSA groups*
}

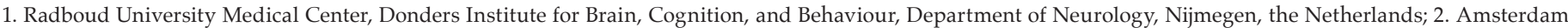

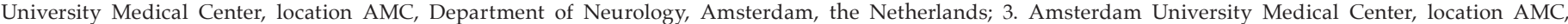

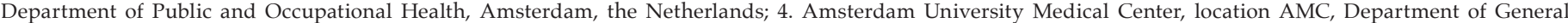

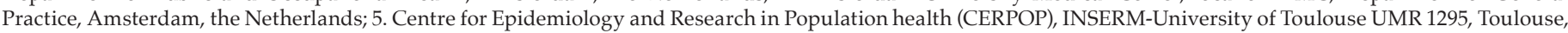

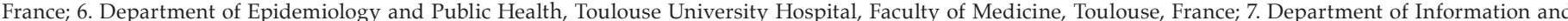

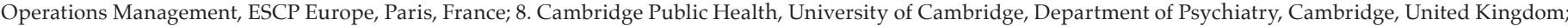
*The members of the preDIVA and MAPT/DSA study groups are listed at the end of the manuscript.

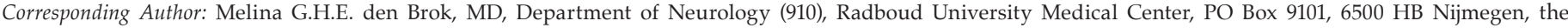
Netherlands, Melina.denBrok@Radboudumc.nl

\begin{abstract}
BACKGROUND: Cardiovascular risk factors and lifestyle factors are associated with an increased risk of cognitive decline and dementia in observational studies, and have been targeted by multidomain interventions.

OBJECTIVES: We pooled individual participant data from two multi-domain intervention trials on cognitive function and symptoms of depression to increase power and facilitate subgroup analyses.

DESIGN: Pooled analysis of individual participant data.

SETTING: Prevention of Dementia by Intensive Vascular Care trial (preDIVA) and Multidomain Alzheimer Preventive Trial
\end{abstract} (MAPT).

PARTICIPANTS: Community-dwelling individuals, free from dementia at baseline.

INTERVENTION: Multidomain interventions focused on cardiovascular and lifestyle related risk factors.

MEASUREMENTS: Data on cognitive functioning, depressive symptoms and apathy were collected at baseline, 2 years and 3-4 years of follow-up as available per study. We analyzed crude scores with linear mixed models for overall cognitive function (Mini Mental State Examination [MMSE]), and symptoms of depression and apathy (15-item Geriatric Depression Scale). Prespecified subgroup analyses were performed for sex, educational level, baseline MMSE $<26$, history of hypertension, and history of stroke, myocardial infarction and/or diabetes mellitus.

RESULTS: We included 4162 individuals (median age 74 years, IQR 72, 76) with a median follow-up duration of 3.7 years (IQR 3.0 to 4.1 years). No differences between intervention and control groups were observed on change in cognitive functioning scores and symptoms of depression and apathy scores in the pooled study population. The MMSE declined less in the intervention groups in those with MMSE $<26$ at baseline ( $\mathrm{N}=250 ; \mathrm{MD}: 0.84 ; 95 \% \mathrm{CI}: 0.15$ to $1.54 ; \mathrm{p}<0.001)$.

CONCLUSIONS: We found no conclusive evidence that multidomain interventions reduce the risk of global cognitive decline, symptoms of depression or apathy in a mixed older population. Our results suggest that these interventions may be more effective in those with lower baseline cognitive functioning. Extended follow-up for dementia occurrence is important to inform on the potential long-term effects of multidomain interventions.

Key words: Multidomain intervention trials, cognition, depression, apathy, pooled analysis.

\section{Introduction}

The global prevalence of dementia is expected to triple in the coming decades. Over 50 million individuals were living with dementia in 2019, and this number might rise to 152 million by 2050 (1). Around $30-40 \%$ of dementia cases might be attributable to potentially modifiable risk factors such as midlife hypertension, depression and physical inactivity (2-4). However, evidence from randomized controlled trials targeting these risk factors is inconsistent $(5,6)$.

Several large multidomain intervention studies using cognitive functioning or dementia as primary outcome have been performed in older persons from the general population free from dementia at baseline (7-9). Two major trials, the prevention of dementia by intensive vascular care trial (preDIVA) and Multidomain Alzheimer Preventive Trial (MAPT) reported no significant effect on their respective primary outcomes dementia and cognitive decline $(10,11)$. The Finnish Geriatric Intervention Study to Prevent Cognitive Impairment and Disability trial (FINGER) is the only study so far which reported a modest excess improvement of cognitive functioning in the intervention group compared to an improvement in the control group (12).

Depression is a potentially modifiable risk factor for 


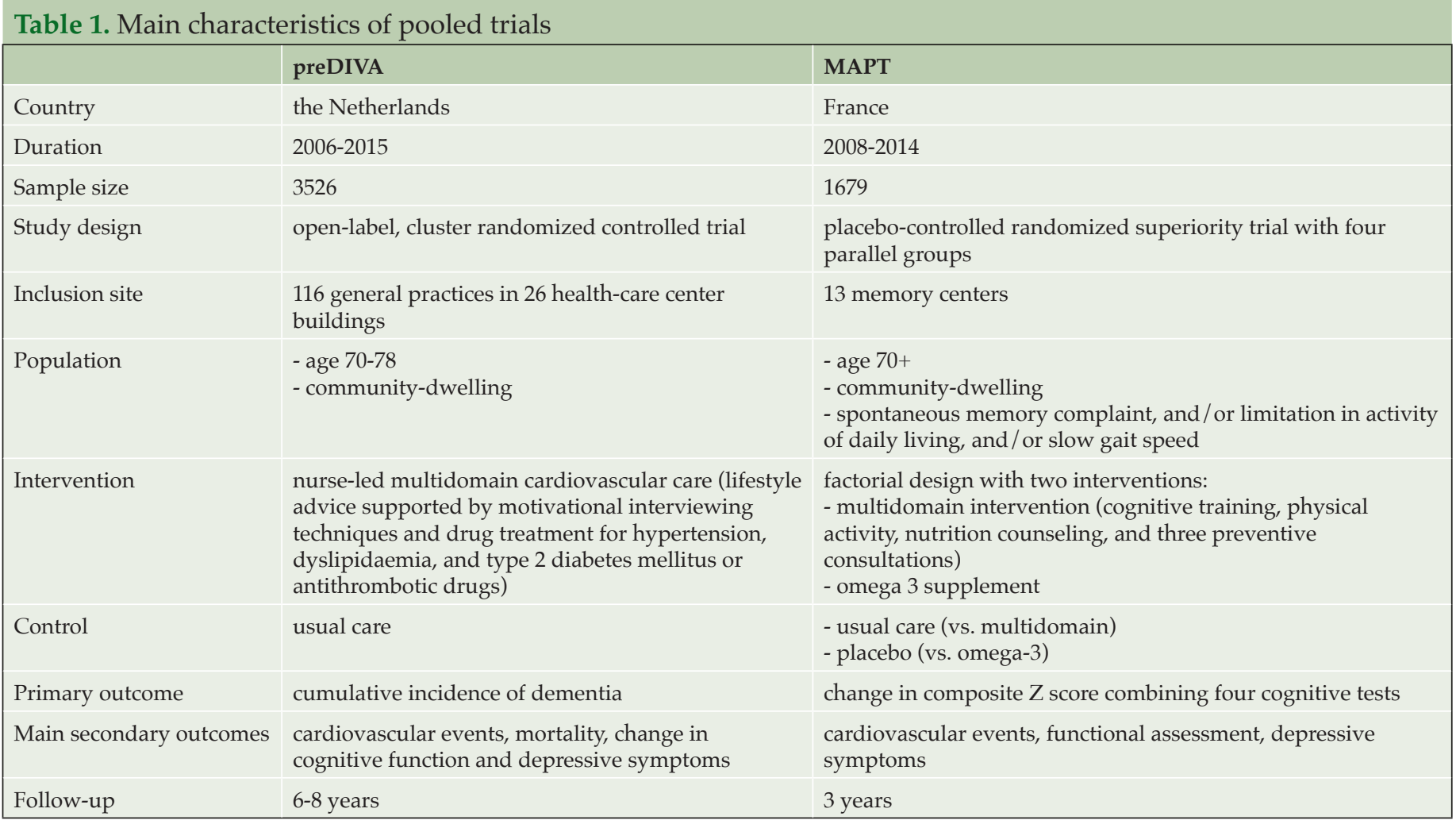

dementia (3), and there is a bidirectional association between depression and cardiovascular risk factors and events (13-17). Apathy is associated with cardiovascular risk factors and increased risk of dementia $(18,19)$. A multidomain intervention targeting the same risk factors as those for dementia, could potentially reduce depressive symptoms and apathy. However, none of the trials reported significant effects of the interventions on symptoms of depression, and apathy was not reported.

Individual multidomain trials may have suffered from lack of power, due to the improvement in those in the control condition. In the preDIVA study, subgroup analyses suggested a potential beneficial effect in those with untreated hypertension, those with no history of cardiovascular disease at baseline, and in males (10). A more personalized approach, with interventions tailored to specific subgroups, could potentially lead to better intervention effects than a one-size-fits-all approach.

We pooled individual participant data from two large multidomain intervention trials targeting cardiovascular and lifestyle related risk factors to increase power and allow for subgroup analyses to detect possible intervention effects on cognition, symptoms of depression and apathy.

\section{Methods}

\section{Study design and participants}

We combined individual participant data from two large multidomain intervention trials targeting vascular and lifestyle-related risk factors in older people $(10,11)$. Both studies were European multicenter randomized controlled trials and included 3526 (preDIVA) and 1679 (MAPT) community dwelling individuals free from dementia at baseline, recruited from either general practices (preDIVA) or memory centers (MAPT). Individuals were aged 70 years or older and participants in MAPT were at increased risk for cognitive decline, operationalized as at least one of the three following criteria: spontaneous memory complaint expressed, limitation in one instrumental activity of daily living, or slow gait speed. The multidomain interventions consisted of individual or group sessions providing lifestyle advice concerning diet, physical activity and vascular risk factors. The MAPT study included cognitive training in the intervention. If needed, participants were advised to contact their general practitioner for optimization or initiation of drug treatment for cardiovascular risk factors. Control groups received usual care $(10,11)$. Main characteristics of the trials are specified in table 1 . Study protocols have been published previously $(20,21)$. The MAPT study had a factorial design, with omega-3 supplementation in an additional arm (but there was no effect of this intervention on the trial's primary or secondary outcomes). In this analysis we only evaluate 
Table 2. Baseline characteristics of the pooled sample

\begin{tabular}{|c|c|c|c|c|}
\hline & $\begin{array}{l}\text { All individuals } \\
\mathrm{N}=4162(100 \%)\end{array}$ & $\begin{array}{c}\text { Control } \\
\mathrm{N}=1996(48.0 \%)\end{array}$ & $\begin{array}{c}\text { Intervention } \\
\mathrm{N}=2166(52.0 \%)\end{array}$ & P-value \\
\hline \multicolumn{5}{|l|}{ Study } \\
\hline - preDIVA & $2818(67.7)$ & $1329(66.6)$ & $1489(68.7)$ & \\
\hline - MAPT & $1344(32.3)$ & $667(33.4)$ & $677(31.3)$ & \\
\hline \multicolumn{5}{|l|}{ Demographics } \\
\hline Age y, median (IQR) & $74(72-76)$ & $74(72-77)$ & $74(72,76)$ & 0.8 \\
\hline Female sex, n $(\%)$ & $2403(57.7)$ & $1159(58.1)$ & $1244(57.4)$ & 0.7 \\
\hline \multicolumn{5}{|l|}{ Educational level, n (\%) } \\
\hline - Low & $922(22.4)$ & $451(23.0)$ & $471(21.9)$ & 0.7 \\
\hline - Intermediate & $2070(50.3)$ & $976(49.7)$ & $1094(50.8)$ & \\
\hline - High & $1125(27.3)$ & $536(27.3)$ & $589(27.3)$ & \\
\hline \multicolumn{5}{|l|}{ Biological risk factors } \\
\hline Systolic blood pressure (mmHg), mean (SD) & $150.3(21.6)$ & $149.3(20.9)$ & $151.2(22.1)$ & 0.006 \\
\hline LDL cholesterol (mmol/L), mean (SD) & $3.1(1.0)$ & $3.2(1.0)$ & $3.1(1.0)$ & 0.003 \\
\hline BMI $\left(\mathrm{kg} / \mathrm{m}^{2}\right)$, mean (SD) & $27.0(4.2)$ & $27.0(4.1)$ & $27.1(4.2)$ & 0.3 \\
\hline
\end{tabular}

All individuals with at least one follow-up visit, and comparison of the intervention and control group. Differences between randomization groups in spite of the large numbers, may partly be due to cluster randomization in preDIVA. P-value for comparison between control and intervention. Abbreviations: BMI: Body Mass Index; IQR: interquartile range; LDL cholesterol: low-density lipoprotein cholesterol; SD: standard deviation

the multidomain intervention. The ethics committees in the respective medical centers approved both trials and all individuals gave written informed consent. Both trials were registered, respectively in the ISRCTN registry (preDIVA: ISRCTN29711771) and on ClinicalTrials.gov (MAPT: NCT00672685).

\section{Outcomes}

Global cognitive function was assessed with the MiniMental State Examination (MMSE). MMSE items were divided into anterograde episodic memory, evaluated with the delayed recall item (item 5, max 3 points) or 'other cognitive functions' (all other items). Both studies used different neuropsychological tests for memory (Visual Association Test (22) in preDIVA and Free and Cued Selective Reminding (23) in MAPT), precluding the possibility to use these more extensive memory tests in our analyses. As measure of subjective memory loss we used question 10 of the 15-item Geriatric Depression Scale (GDS-15): 'Do you feel you have more problems with memory than most?'. Depressive symptoms were quantified using the crude scores of the 15-item Geriatric Depression Scale (GDS-15) and using a dichotomized cutoff (GDS-15 >5: indicative of depression; $\leq 5$ : not indicative of depression). We additionally assessed the effect on apathy, operationalized as the three apathy items from the GDS-15 (GDS-3A), as has previously been shown to be an appropriate screening instrument for symptoms of apathy (24).

\section{Statistical Analysis}

We included participants with at least one followup visit to analyze the effect of the intervention on cognitive decline, symptoms of depression and apathy. Mean differences in change between the intervention and control group were calculated using linear mixed regression models. Crude continuous and binary scores were used as outcome, adjusted for baseline scores of the specific tests using analysis of covariance (ANCOVA). We used follow-up measures of the outcome of interest as outcome and treatment allocation as predictor, including random intercepts for participant and health center level. Model fit was estimated using Akaike Information Criterion values, and more complex models (i.e. with additional random slope for randomization allocation or the time difference between randomization and the follow-up visit) did not result in a better fit. Analyses were adjusted for the baseline measure of the outcome of interest, study, age, sex, time in study and also for LDL cholesterol and systolic blood pressure because of significant differences in baseline values between randomization groups. Furthermore, linear interaction for time in study was assessed by means of an interaction term. Predefined subgroup analyses were performed for 1) sex, since multidomain interventions may have differential effects in men and women, 2) baseline hypertension status defined as history of hypertension and / or systolic blood pressure of $>140 \mathrm{mmHg}$, 3) history of myocardial infarction, stroke and/or diabetes at baseline, since there is a high probability that individuals with a history of cardiovascular disease already receive a 
Table 3. Mean difference in effect of multidomain interventions on cognition, symptoms of depression and apathy after 3-4 years of follow-up

\begin{tabular}{|c|c|c|c|c|c|c|c|}
\hline & \multirow{2}{*}{$\begin{array}{c}\text { Number of } \\
\text { observations in } \\
\text { analysis } \\
\text { N }\end{array}$} & \multirow{2}{*}{$\begin{array}{c}\text { Number of } \\
\text { individuals in } \\
\text { analysis }\end{array}$} & \multicolumn{2}{|c|}{ Baseline mean } & \multicolumn{2}{|c|}{$\begin{array}{l}\text { Mean difference baseline } \\
\text { and follow-up }\end{array}$} & \multirow{2}{*}{$\begin{array}{l}\text { Mean difference } \\
\text { between intervention } \\
\text { and control group } \\
\text { MDc }(95 \% \mathrm{CI})\end{array}$} \\
\hline & & & Control & Intervention & Control & Intervention & \\
\hline \multicolumn{8}{|l|}{ Cognitive functioning } \\
\hline MMSE (range 0-30)* & 6677 & 3704 & 28.26 & 28.25 & -0.09 & -0.05 & $0.03(-0.06$ to 0.13$)$ \\
\hline $\begin{array}{l}\text { Memory (MMSE item 5) } \\
(\text { range } 0-3)^{*}\end{array}$ & 6774 & 3704 & 2.31 & 2.29 & 0.03 & 0.05 & $0.004(-0.04$ to 0.05$)$ \\
\hline Other (range $0-27)^{*}$ & 6677 & 3704 & 25.95 & 25.96 & -0.12 & -0.10 & $0.03(-0.05$ to 0.10$)$ \\
\hline $\begin{array}{l}\text { Subjective memory loss } \\
\text { (GDS15-Q10, \% yes)† }\end{array}$ & 6388 & 3503 & 18.15 & 19.53 & 0.03 & -0.79 & $-0.51(-2.52$ to 1.50$)$ \\
\hline \multicolumn{8}{|l|}{ Depressive symptoms } \\
\hline GDS (range $0-15) \dagger$ & 6279 & 3506 & 2.05 & 1.96 & 0.22 & 0.21 & $-0.04(-0.16$ to 0.07$)$ \\
\hline $\begin{array}{l}\text { Depressive symptoms }(\% \\
\text { GDS }>5)+\end{array}$ & 6279 & 3506 & 8.93 & 8.04 & 2.59 & 2.54 & $-0.68(-2.32$ to 0.97$)$ \\
\hline Apathy (range $0-3)+$ & 6325 & 3503 & 0.70 & 0.69 & 0.12 & 0.12 & $-0.007(-0.05$ to 0.04$)$ \\
\hline
\end{tabular}

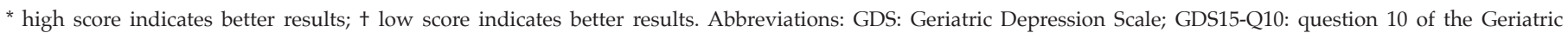

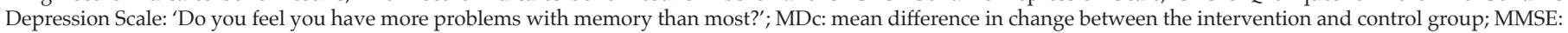
Mini-Mental State Examination; 95\% CI: 95\% confidence interval

form of intervention and therefore, there might be more room for improvement in participants without a history of cardiovascular disease, 4) baseline MMSE <26, since individuals with lower baseline cognitive functioning might benefit more from a multidomain intervention, 5) educational level (low and high) because this is an important risk factor for dementia and is also associated with low socioeconomic status (3). Additional analyses were performed by study, to assess heterogeneity between the different studies. A p-value for interaction $<0.05$ was considered to reflect a significant interaction. Post-hoc analyses included exploration of differential dropout by comparing baseline characteristics of individuals included in the current study to individuals without any follow-up visits, and subgroup analyses for different cutoff values of baseline MMSE. Analyses were conducted in Rstudio (version 3.6.1, package "lme4") (25).

\section{Results}

Of a total of 5205 individuals in the two studies, 4162 $(80 \%)$ individuals had at least one follow-up visit and were included in the present analysis (median follow-up duration: 3.7 years; IQR 3.0 to 4.14 years). The median age at baseline was 74 years (IQR 72, 76 years) and slightly more women were included $(57.7 \%)$. No significant between-group differences in baseline characteristics were found in the pooled population, except for mean systolic blood pressure (control 149.3, SD 20.9; intervention 151.2, SD 22.1; $\mathrm{p}=0.006$ ) and mean LDL cholesterol (control 3.19, SD 0.97; intervention 3.10, SD 0.95; $\mathrm{p}=0.003$ ) (Table 2). eTable 1 shows baseline characteristics of included individuals per study. Participants who did not have a follow-up visit were slightly older, had a lower educational level, a slightly lower MMSE score, and a higher mean systolic blood pressure (eTable 2).

There were no differences in change from baseline to 3-4 year follow-up in MMSE and GDS scores between the control and intervention groups: Total MMSE score deteriorated by 0.09 points in the control group versus 0.05 points in the intervention group (mean difference in change $[\mathrm{MDc}$ ] between intervention and control group: $0.03 ; 95 \%$ confidence interval $[95 \% \mathrm{CI}]-0.06$ to 0.13$)$. Total GDS score deteriorated by 0.21 points in the intervention group versus 0.22 points in the control group (MDc between intervention and control group: $-0.04,95 \% \mathrm{CI}$ -0.16 to 0.07$)$. The GDS-apathy score deteriorated by 0.12 points in both randomization groups (MDc between intervention and control group: -0.007 (-0.05 to 0.04). There was no time by treatment interaction for any of the outcome variables.

\section{Subgroup Analysis}

In individuals with a baseline MMSE score $<26$, total MMSE score improved over time in both randomization groups, but more in the intervention group (MDc 0.84 points, $95 \%$ CI 0.15 to 1.54 ). Similar effects were seen for anterograde episodic memory (MDc 0.21, 95\% CI -0.01 to 0.43 ) and other MMSE items (MDc $0.74,95 \% \mathrm{CI}$ 0.26 to 1.21 ). In those with a baseline MMSE score $\geq 26$, some deterioration or no change in MMSE scores (total, memory or other items) was seen with similar effects in both randomization groups (Table 5, eTable 3 ). No significant differences between randomization groups in change in test scores for cognitive functioning, depressive symptoms or apathy were found for sex, educational level, baseline hypertension status and history of cardiovascular disease or diabetes at baseline (Table 4, Table 5). In MAPT, stronger deterioration on other 

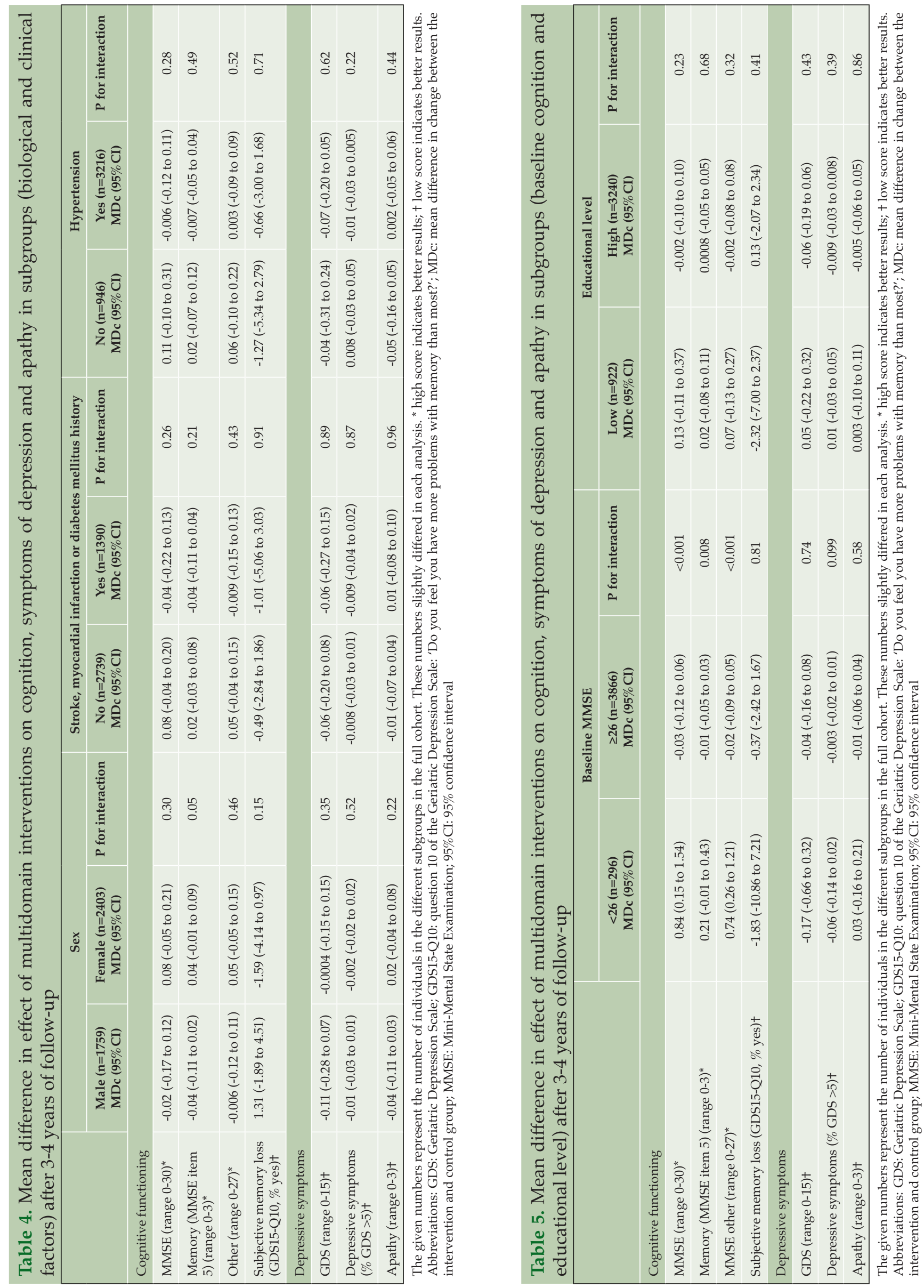
MMSE items was seen in the control group compared to the intervention group, but there was no effect in preDIVA. None of the other outcomes were significantly different between both studies (eTable 4).

To assess the consistency of the effects favoring the intervention in individuals with a low baseline MMSE, we performed post-hoc subgroup analyses stratified for various baseline MMSE cutoff scores (eTable 5). The highest MDc were found with a baseline MMSE cutoff score $<26$, and with increasing baseline MMSE scores, MDc between the intervention and control group gradually decreased. Individuals with MMSE score $<26$ at baseline were significantly lower educated and had a higher Body Mass Index at baseline (eTable 6). Additional adjustment for these covariates did not significantly change the MDc between the intervention and control group in total MMSE score (baseline MMSE $\leq 26$ : MMSE MDc $0.77,95 \%$ CI 0.07 to $1.47 ; \mathrm{p}<0.001)$. There was no differential dropout between both randomization groups in individuals with a baseline MMSE score $<26$ and $>26$ points.

\section{Discussion}

This pooled analysis of two large randomized controlled trials in community-dwelling individuals over 60 years old did not show an overall effect of multidomain interventions on cognitive function or symptoms of depression or apathy after 3-4 years follow-up. Subgroup analyses suggests that multidomain interventions may improve cognition in those with lower cognitive scores at baseline. We observed no interaction of the effect of the interventions with sex, history of stroke, diabetes mellitus and/or myocardial infarction, hypertension and educational level.

\section{Strengths and limitations}

The major strength of this study is that we pooled data on individual participant level from two large randomized controlled trials, providing more power to detect possible intervention effects on cognition, symptoms of depression and apathy, and to better allow for subgroup analyses to explore whether interventions may be more effective in specific subgroups. Furthermore, variation in inclusion criteria and multidomain interventions between the different trials improved the external validity of our overall results.

Several limitations should be noted. First of all, the use of the MMSE as outcome measure for cognitive studies has limitations. This time-honored test was designed as a cognitive screening test and does not measure cognitive function as comprehensively or sensitively, or with such detailed quantification, as a full neuropsychological evaluation does. Assessments using cognitive screening instruments such as the MMSE are known to show substantial variation over time, depending on conditions such as e.g. illness, stress or sleep deprivation, particularly in people without cognitive impairments (26), although the same holds for full neuropsychological evaluation. A possible random error caused by these fluctuations may have resulted in bias towards the null. Another disadvantage of the MMSE in populations such as under study here, is its ceiling effect. It lacks the ability to differentiate well between healthy individuals and early signs of dementia in individuals with MMSE scores in the range of 24-30. However, despite its limitations, as with any other screening test, the MMSE is characterized by an unprecedented dissemination and appreciation among the scientific epidemiological and dementia community. Moreover, these interventions were not designed to boost cognition, but to prevent cognitive decline. Less decline in MMSE was a more likely hypothesis than more increment in MMSE due to the intervention - nuancing the potential ceiling effect. Secondly, attrition bias could have influenced our results, since those who dropped out were significantly older, had a lower educational level, higher mean systolic blood pressure, and lower MMSE scores; i.e. they were at higher risk of cognitive decline. This could have biased our results to the null, since those with lower cognitive function appear to potentially benefit most from intervention. Lastly, while both studies were designed to test the efficacy of multidomain interventions in elderly, there were important differences in study design and populations between both trials which could have impacted the results of this study. The recruitment strategy of individuals in preDIVA was population-based through general practices, whereas MAPT recruited individuals at risk for cognitive decline through memory centers. Cognitive training was not part of the intervention in preDIVA, but the intervention in MAPT strongly focused on cognitive training in the intervention group, which was given in supervised sessions, in contrast to the physical activity and nutrition components which were simply based on advice. Moreover, cardiovascular risk factors in the intervention group in preDIVA were assessed every four months by a practice nurse, and if necessary, medical interventions such as drug treatment were advised according to a detailed protocol. The MAPT intervention assessed cardiovascular risk factors annually, and there was less focus on drug treatment. These differences might improve the external validity of our results, but they are likely to also cause heterogeneity. However, there were no structural differences in intervention effects between the MAPT and preDIVA intervention.

Although overall this study did not show beneficial effects of multidomain interventions on symptoms of depression and apathy and cognitive function, several methodological challenges associated with dementia prevention trials complicate the interpretation of these results. First, the development of cognitive impairment and dementia is a slow and insidious process, and risk factors in midlife have a stronger association with 
incident dementia than risk factors in later life (27-30). However, since inclusion of participants in midlife requires an unrealistically long follow-up (31), these trials included participants in later life, far beyond the stage of life in which interventions are expected to have their optimal effect. Second, follow-up durations for the current analyses were too short to detect an effect on incident dementia, as most clear clinically relevant outcome. Long-term interventions starting at a younger age might be needed to achieve clinically relevant effects.

Although observational studies have shown consistent evidence supporting the association of cardiovascular risk factors and lifestyle related factors with cognitive decline and dementia, and some interventions targeting these factors have shown to improve cognitive function $(3,4,32)$, trials evaluating multidomain interventions report inconsistent results $(10-12,33)$. Variation in intensity, components of the multidomain intervention, and characteristics of the control condition in the individual trials may partly explain these inconsistent results. Alternative explanations include that the associations found in observational studies do not reflect a causal relationship or that the harmful effects have been effectuated by the time these interventions started.

Subgroup analyses for individuals with low baseline MMSE score yielded consistent results with beneficial effects of the multidomain intervention on cognitive function: total MMSE score, including anterograde episodic memory and other MMSE items increased more in the intervention group. Similar effects on memory were seen in a multidomain intervention trial in black individuals with MCI (34). The size of these groups (MMSE <26) was small within the overall studies $(n=296)$. However, the gradual decrease in MDc with increasing baseline MMSE score appeared to be consistent (Table 4 ), and the results did not change with additional adjustment for differences in baseline characteristics. Furthermore, results were still statistically significant after multiple-comparison correction $(p<0.01$ after Bonferroni correction). These findings should be confirmed in the multidomain intervention trials that are currently underway (35-37).

The underlying etiology of dementia is heterogeneous, and a multidomain intervention tailored to specific subgroups could potentially lead to better intervention effects than a one-size-fits-all approach. The results of this study, showing that, in later life, this type of intervention may be more effective in those with lower baseline cognitive scores, should be considered in the design of future dementia prevention trials.

\section{Conclusion}

This study with pooled data at the individual participant level from two large, randomized controlled trials did not show conclusive evidence that multidomain interventions can reduce the risk of cognitive decline or symptoms of depression and apathy in a mixed older population. These interventions may be more effective in those with lower baseline cognitive function. Extended follow-up for dementia outcomes is important to evaluate whether multidomain interventions can indeed have beneficial effects crossing the threshold of minimal clinically important difference. These extended followups are planned and ongoing in both trials.

Funding: The present study was financially supported by the European Union's Seventh Framework Program (FP7, 2007- 2013, grant nr. 305374), as part of the HATICE project. The preDIVA study was financially supported by the Dutch Ministry of Health, Welfare and Sport (grant number 50-50110-98-020), the Dutch Innovation Fund of Collaborative Health Insurances (grant number 05-234), and the Netherlands Organisation for Health Research and Development (grant number 62000015). The MAPT study was supported by grants from the Gérontopôle of Toulouse, the French Ministry of Health (PHRC 2008, 2009), Pierre Fabre Research Institute (manufacturer of the omega-3 supplement), ExonHit Therapeutics SA, and Avid Radiopharmaceuticals Inc. The promotion of this study was supported by the University Hospital Center of Toulouse. The data sharing activity was supported by the Association Monegasque pour la Recherche sur la maladie d'Alzheimer (AMPA) and the UMR 1027 Unit INSERM-University of Toulouse III. The sponsors had no role in the design and conduct of the study; in the collection, analysis, and interpretation of data; in the preparation of the manuscript; or in the review or approval of the manuscript.

Acknowledgements: The authors would like to thank all the participants to the preDIVA and MAPT studies, and the members of the preDIVA and MAPT trial teams for their help in data collection and management.

Declaration of Helsinki: The studies included comply with the Declaration of Helsinki, locally appointed ethics committees have approved the research protocols and informed consent has been obtained from all the subjects.

Conflict of interest disclosure: M.G.H.E. den Brok, M.P. Hoevenaar-Blom, N. Coley, J. van Dalen, C. Brayne, W.A. van Gool, E.P. Moll van Charante, E. Richard have nothing to disclose. Y. Meiller, J. Guillemont and S. Andrieu report grants from the European Union, during the conduct of the study (FP7, 2007-2013, grant nr. 305374). S. Andrieu additionally reports grants from the French Ministry of health (PHRC 2008, 2009), Pierre Fabre Research Institute, and AMPA during the conduct of the study.

Study group: The members of the preDIVA study group: Eric P. Moll van Charante, Edo Richard, Lisa S. Eurelings, Jan-Willem van Dalen, Suzanne A. Ligthart, Emma F. van Bussel, Marieke P. Hoevenaar-Blom, Marinus Vermeulen, Willem A. van Gool (Amsterdam University Medical Center, location AMC Amsterdam, the Netherlands). The members of the MAPT/DSA study group: MAPT study group: Principal investigator: Bruno Vellas (Toulouse); Coordination: Sophie Guyonnet; Project leader: Isabelle Carrié; CRA: Lauréane Brigitte; Investigators: Catherine Faisant, Françoise Lala, Julien Delrieu, Hélène Villars; Psychologists: Emeline Combrouze, Carole Badufle, Audrey Zueras; Methodology, statistical analysis, and data management: Sandrine Andrieu, Christelle Cantet, Christophe Morin; Multidomain group: Gabor Abellan Van Kan, Charlotte Dupuy, Yves Rolland (physical and nutritional components), Céline Caillaud, Pierre-Jean Ousset (cognitive component), Françoise Lala (preventive consultation). The cognitive component was designed in collaboration with Sherry Willis from the University of Seattle, and Sylvie Belleville, Brigitte Gilbert, and Francine Fontaine from the University of Montreal. Coinvestigators in associated centers: JeanFrançois Dartigues, Isabelle Marcet, Fleur Delva, Alexandra Foubert, Sandrine Cerda (Bordeaux); Marie-Noëlle Cuffi, Corinne Costes (Castres); Olivier Rouaud, Patrick Manckoundia, Valérie Quipourt, Sophie Marilier, Evelyne Franon (Dijon); Lawrence Bories, Marie-Laure Pader, Marie-France Basset, Bruno Lapoujade, Valérie Faure, Michael Li Yung Tong, Christine Malick-Loiseau, Evelyne CazabanCampistron (Foix); Françoise Desclaux, Colette Blatge (Lavaur); Thierry Dantoine, Cécile Laubarie-Mouret, Isabelle Saulnier, Jean-Pierre Clément, Marie-Agnès Picat, Laurence Bernard-Bourzeix, Stéphanie Willebois, Iléana Désormais, Noëlle Cardinaud (Limoges); Marc Bonnefoy, Pierre Livet, Pascale Rebaudet, Claire Gédéon, Catherine Burdet, Flavien Terracol (Lyon), Alain Pesce, Stéphanie Roth, Sylvie Chaillou, Sandrine Louchart (Monaco); Kristel Sudres, Nicolas Lebrun, Nadège Barro-Belaygues (Montauban); Jacques Touchon, Karim Bennys, Audrey Gabelle, Aurélia Romano, Lynda Touati, Cécilia Marelli, Cécile Pays (Montpellier); Philippe Robert, Franck Le Duff, Claire Gervais, Sébastien Gonfrier (Nice); Yannick Gasnier and Serge Bordes, Danièle Begorre, Christian Carpuat, Khaled Khales, Jean-François Lefebvre, Samira Misbah El Idrissi, Pierre Skolil, Jean-Pierre Salles (Tarbes). Biological sample collection: Bertrand Perret, Claire Vinel, Sylvie CasparBauguil (Toulouse). Safety management: Pascale Olivier-Abbal. DSA Group: Sandrine Andrieu, Christelle Cantet, Nicola Coley. 
Open Access: This article is distributed under the terms of the Creative Commons Attribution 4.0 International License (http:/ / creativecommons.org/ licenses/by/4.0/), which permits use, duplication, adaptation, distribution and reproduction in any medium or format, as long as you give appropriate credit to the original author(s) and the source, provide a link to the Creative Commons license and indicate if changes were made.

\section{References}

1. Alzheimer's Disease International (ADI). World Alzheimer Report 2019: Attitudes to Dementia.; 2019. https://www.alzint.org/u/ WorldAlzheimerReport2019.pdf. Accessed 07-12-2020

2. Livingston G, Sommerlad A, Orgeta V, et al. Dementia prevention, intervention, and care. Lancet. 2017;390(10113):2673-734 http:/ / dx.doi. org/10.1016/S0140-6736(17)31363-6.

3. Livingston G, Huntley J, Sommerlad A, et al. Dementia prevention, intervention, and care: 2020 report of the Lancet Commission. Lancet 2020;396(10248):413-46 https: / doi.org/10.1016/S0140-6736(20)30367-6.

4. Norton S, Matthews FE, Barnes DE, Yaffe K, Brayne C. Potential for primary prevention of Alzheimer's disease: An analysis of populationbased data. Lancet Neurol. 2014;13(8):788-94 https: / / doi.org/10.1016/S14744422(14)70136-X.

5. Coley N, Andrieu S, Gardette V, et al. Dementia prevention: Methodological explanations for inconsistent results. Epidemiol Rev. 2008;30(1):35-66 https: / / doi.org/10.1093/epirev/mxn010.

6. Yaffe K. Modifiable risk factors and prevention of dementia what is the latest evidence? JAMA Intern Med. 2018;178(2):281-2 https:// doi.org/10.1001/ jamainternmed.2017.7299.

7. Espeland MA, Luchsinger JA, Baker LD, et al. Effect of a long-term intensive lifestyle intervention on prevalence of cognitive impairment. Neurology. 2017;88(21):2026-35 https:/ / doi.org/10.1212/WNL.0000000000003955.

8. Chen LK, Hwang AC, Lee WJ, et al. Efficacy of multidomain interventions to improve physical frailty, depression and cognition: data from clusterrandomized controlled trials. J Cachexia Sarcopenia Muscle. 2020;11(3):650-62 https: / / doi.org/10.1002/jcsm.12534.

9. Hafdi M, Hoevenaar-Blom MP, Richard E. Multi-domain interventions for the prevention of dementia and cognitive decline (Protocol). Cochrane Database Syst Rev. 2020;(4) https: / / doi.org/10.1002/14651858.CD013572.

10. Moll van Charante E, Richard E, Eurelings LS, et al. Effectiveness of a 6-year multidomain vascular care intervention to prevent dementia (preDIVA): a cluster-randomised controlled trial. Lancet. 2016;388(10046):797-805 https:/ / doi.org/10.1016/S0140-6736(16)30950-3.

11. Andrieu S, Guyonnet S, Coley N, et al. Effect of long-term omega 3 polyunsaturated fatty acid supplementation with or without multidomain intervention on cognitive function in elderly adults with memory complaints (MAPT): a randomised, placebo-controlled trial. Lancet Neurol. 2017;16(5):377-89 https: / / doi.org/10.1016/S1474-4422(17)30040-6.

12. Ngandu T, Lehtisalo J, Solomon A, et al. A 2 year multidomain intervention of diet, exercise, cognitive training, and vascular risk monitoring versus control to prevent cognitive decline in at-risk elderly people (FINGER): a randomised controlled trial. Lancet (London, England). 2015 Jun;385(9984):2255-63 https: / / doi.org/10.1016/S0140-6736(15)60461-5.

13. Anderson RJ, Freedland KE, Clouse RE, Lustman PJ. The Prevalence of Comorbid Depression in Adults with Diabetes. Diabetes Care. 2001;24(6):1069 78 https: / / doi.org/10.2337 / diacare.24.6.1069.

14. Gan Y, Gong Y, Tong X, et al. Depression and the risk of coronary heart disease: A meta-analysis of prospective cohort studies. BMC Psychiatry. 2014;14(1):1-11 https: / / doi.org/10.1186/s12888-014-0371-z.

15. Mezuk B, Eaton WW, Albrecht S, Golden SH. Depression and type 2 diabetes over the lifespan: A meta-analysis. Diabetes Care. 2008;31(12):2383-90 https: / / doi.org/10.2337/dc08-0985.

16. Meng L, Chen D, Yang Y, Zheng Y, Hui R. Depression increases the risk of hypertension incidence: A meta-analysis of prospective cohort studies. J Hypertens. 2012;30(5):842-51 https://doi.org/10.1097/ HJH.0b013e32835080b7

17. Wu Q, Kling JM. Depression and the Risk of Myocardial Infarction and Coronary Death. Med (United States). 2016;95(6):1-9 https: / / doi.org/10.1097/ MD.0000000000002815.

18. Ligthart SA, Richard E, Fransen NL, et al. Association of vascular factors with apathy in community-dwelling elderly individuals. Arch Gen Psychiatry. 2012;69(6):636-42 https: / / doi.org/10.1001/archgenpsychiatry.2011.1858.
19. Van Dalen JW, Van Wanrooij LL, Moll Van Charante EP, Brayne C, Van Gool WA, Richard E. Association of Apathy with Risk of Incident Dementia: A Systematic Review and Meta-analysis. JAMA Psychiatry. 2018;75(10):1012-21 https:/ / doi.org/10.1001/jamapsychiatry.2018.1877.

20. Richard E, Den Heuvel E Van, Moll Van Charante EP, et al. Prevention of dementia by intensive vascular care (prediva): A cluster-randomized trial in progress. Alzheimer Dis Assoc Disord. 2009;23(3):198-204 https:/ / doi org/10.1001/jamapsychiatry.2018.1877.

21. Vellas B, Carrie I, Gillette-Guyonnet S, et al. MAPT study: a multidomain approach for preventing Alzheimer's disease: design and baseline data. J Prev Alzheimers Dis. 2014;1(1):13-22 http:/ / dx.doi.org/10.14283/jpad.2014.34.

22. Lindeboom J, Schmand B, Tulner L, Walstra G, Jonker C. Visual association test to detect early dementia of the Alzheimer type. J Neurol Neurosurg Psychiatry. 2002;73(2):126-33 https: / / doi.org/10.1136/jnnp.73.2.126.

23. Grober E, Buschke H, Crystal H, Bang S, Dresner R. Screening for dementia by memory testing. Neurology. 1988;38(6):900-3 https:// doi.org/10.1212/ wnl.38.6.900.

24. van der Mast RC, Vinkers DJ, Stek ML, et al. Vascular disease and apathy in old age. The Leiden 85-plus Study. Int J Geriatr Psychiatry. 2008;23(3):266-71 https: / / doi.org/10.1002/gps.1872.

25. Bates D, Mächler M, Bolker B, Walker S. Fitting Linear Mixed-Effects Model Using lme4. J Stat Softw. 2015;67(1):1-48 https:/ / doi.org/10.18637/jss.v067. i01.

26. Richard E, Brayne C. Mild cognitive impairment - not always what it seems. Nat Rev Neurol. 2014;10(3):130-1 https:/ / doi.org/10.1038/nrneurol.2014.23.

27. Kivimäki M, Luukkonen R, Batty GD, et al. Body mass index and risk of dementia: Analysis of individual-level data from 1.3 million individuals. Alzheimer's Dement. 2018;14(5):601-9 https://doi.org/10.1016/j. jalz.2017.09.016.

28. Pedditizi E, Peters R, Beckett N. The risk of overweight/obesity in mid-life and late life for the development of dementia: A systematic review and metaanalysis of longitudinal studies. Age Ageing. 2016;45(1):14-21 https:// doi. org/10.1093/ageing/afv151.

29. Abell JG, Kivimäki M, Dugravot A, et al. Association between systolic blood pressure and dementia in theWhitehall II cohort study: Role of age, duration and threshold used to define hypertension. Eur Heart J. 2018:39(33):3119-25 https: / / doi.org/10.1093/ eurheartj/ehy288.

30. McGrath ER, Seshadri S. Author response: Blood pressure from mid- to late life and risk of incident dementia. Neurology. 2018;91(3):149 https:/ / doi org/10.1212/WNL.0000000000005810.

31. Richard E, Andrieu S, Solomon A, et al. Methodological challenges in designing dementia prevention trials - The European Dementia Prevention Initiative (EDPI). J Neurol Sci [Internet]. 2012;322(1-2):64-70. Available from: http:/ / dx.doi.org/10.1016/j.jns.2012.06.012

32. Andrieu S, Coley N, Lovestone S, Aisen PS, Vellas B. Prevention of sporadic Alzheimer's disease: Lessons learned from clinical trials and future directions. Lancet Neurol. 2015;14(9):926-44 https:// doi.org/10.1016/S14744422(15)00153-2.

33. Richard E, Moll van Charante EP, Hoevenaar-Blom MP, et al. Healthy ageing through internet counselling in the elderly (HATICE): a multinational, randomised controlled trial. Lancet Digit Heal. 2019;1(8):e424-34 33. https:/ / doi.org/10.1016/S2589-7500(19)30153-0.

34. Rovner BW, Casten RJ, Hegel MT, Leiby B. Preventing Cognitive Decline in Black Individuals With Mild Cognitive Impairment: A Randomized Clinical Trial. JAMA Neurol. 2018;75(12):1487-1493 https:/ / doi.org/10.1001/ jamaneurol.2018.2513

35. PRODEMOS consortium. Prevention of Dementia using Mobile Phone Applications (PRODEMOS). https:/ / www.prodemos-project.eu/. Accessed 16-02-2021.

36. Rosenberg A, Mangialasche F, Ngandu T, Solomon A, Kivipelto M. Multidomain Interventions to Prevent Cognitive Impairment, Alzheimer's Disease, and Dementia: From FINGER to World-Wide FINGERS. J Prev Alzheimer's Dis. 2020;7(1):29-36 https:/ / doi.org/10.14283/jpad.2019.41.

37. de Souto Barreto P, Pothier K, Soriano G, et al. A Web-Based Multidomain Lifestyle Intervention for Older Adults: The eMIND Randomized Controlled Trial. J Prev Alzheimer's Dis. 2021;8(2):142-50 https:// doi.org/10.14283/ jpad.2020.70

How to cite this article: M.G.H.E. den Brok, M.P. Hoevenaar-Blom, N. Coley, et al. The Effect of Multidomain Interventions on Global Cognition, Symptoms of Depression and Apathy - A Pooled Analysis of Two Randomized Controlled Trials. J Prev Alz Dis 2022;1(9):96-103; http:/ / dx.doi.org/10.14283/jpad.2021.53 